\title{
Effect of instructor's actions and attitudes on student's motivation and discussion process in TBL class for graduate students
}

\author{
Shotaro Imai *, Ankit A. Ravankar ${ }^{\dagger}$, \\ Michiyo Shimamura *, Taichi E. Takasuka \\ Go Chiba ${ }^{\dagger}$ Yasuhiro Yamanaka ${ }^{*}$
}

\begin{abstract}
Hokkaido University launched a new trans-graduate school education program "Nitobe School" in 2015 to develop students to achieve high-level transferable skills as well as to enhance their advanced specialty. In this paper, we report the case study of the effect of teaching actions and attitudes on student's motivation and discussion for trans-graduate education in team-basedlearning (TBL) style class. Since a TBL style class for graduate students who come from different faculties and departments is a new education style, the standard way of instruction needs to be established. Instructors are required to develop effective and efficient methods to educate students. To instruct interdisciplinary TBL class is considered as a faculty development for young instructors since it requires a new ability for applying their experience to supervise groups. We took place questionnaire surveys for both students and instructors to review their class organization and teaching approaches after each quarter. The questionnaire mainly followed teacher's actions during the class. From our study, we concluded that the teacher's actions and attitude had enough influence on the students. We further discuss a suitable number of assigned instructors in such TBL class according to the result and conclude at least two to supervise groups is better.
\end{abstract}

Keywords: Faculty development, Teaching actions and attitudes, Team-based learning, Transgraduate school education

\section{Introduction}

Graduate school education is traditionally considered as the place to cultivate specialists who have high-level and reliable abilities, skills and expertise. The School Education Law in Japan lays down "Graduate Schools are designed to teach and study academic theories and

\footnotetext{
* Institute for the Advancement of Higher Education, Hokkaido University, Japan

Research Faculty of Engineering, Hokkaido University, Japan

$\$$ Innovation Promotion Office via Marine-Earth Science and Technology, Japan Agency for Marine-Earth

Science and Technology (JAMSTEC), Japan

$\S \quad$ Research Faculty of Agriculture, Hokkaido University, Japan

** Research Faculty of Environmental Science, Hokkaido University, Japan
} 
applications, and inquire deeply into them or cultivate knowledge and outstanding ability in depth to engage in professions which require advanced expertise, while contributing to the development of culture" in Paragraph 1 of Article 99 [1]. Graduate school education in Japan traditionally adopts a kind of apprentice system to nurture a specialist in a specific research field. Graduate students usually learn the expertise and establish their research style by their supervisors through the research work in individual laboratory. In this sense, education of graduate students entrusts their supervisors. Therefore, know-how and approaches for graduate school education are not shared and established.

Recent higher education, however, is demanded to nurture transferable skills and knowledge, such as "Key competencies" [2], "Gakushi-ryoku" [3] and "Fundamental Competencies for Working Persons" [4] to correspond to a global society. The graduate school education is also expected to develop students to achieve advanced level competencies based on their specialties and abilities to play a leading role in the international and multidisciplinary societies. The central council for education in Japan published the report [5] in 2005, in which they stated the functions of cultivation of human resources in graduate school education to correspond a knowledge-based society.

To correspond the demand, the central council for education in Japan also stated the significance of faculty development (FD) and an evaluation and assessment methods of learning outcomes for graduate education in [6]. Reference [7] introduced handbooks of supervision in graduate school published in foreign countries and [8] reported the experiences of Ph.D. students in foreign universities. Discussion on graduate school education in Japan to correspond the recent society is an important topic of higher education.

In 2014, Hokkaido University planned out "Future Strategy for the 150th Anniversary of Hokkaido University" [9], in which the educational strategy is described that "Hokkaido University will produce graduates who will play a leading role in contributing to the development of the global society. As specialists in their respective fields, they will possess sound judgment and deep insight, along with the ability to understand and communicate with different cultures." Hokkaido University launched the new trans-graduate school program "Nitobe School" [10] in 2015 as one of main educational components of "Top Global University Project" organized by the Ministry of Education, Culture, Sports, Science and Technology (MEXT), Japan [11]. The concept and systems of the School are reported in [12]. The program aims to develop generic competencies of the graduate students beside their home graduate and professional school programs. The program also gives opportunities to work with students from various backgrounds though Team-based-learning (TBL) and Project-based-learning (PBL) style classes. The school also aims that this new education program will train not only students but young faculty members. It is expected that the young faculties will learn a new approach to educate graduate students. To develop a teaching ability of young faculty, several instructors are assigned in one class to supervise students and share each teaching approach.

One of the challenging issues of Nitobe School is the education method of graduate school classes in TBL/PBL style. The role of instructors in TBL/PBL style class especially for trans-graduate school education is not established yet. While the relationship between graduate students and supervisors in university is traditionally considered as apprentices and masters, the TBL/PBL style class in trans-graduate school education requires a new relationship. Instructors in TBL/PBL style class are usually considered as facilitators or advisors. They do not influence the group discussion in any way, but should make sure that the discussions are progressing in the right direction. Since the students who join Nitobe School come from different home graduate program or professional program, they do not 
share common background knowledge, languages, and friendship. Thus, we should establish a new method of TBL/PBL style classes for graduate students to become specialists as well as generalists to be able to play a leading role in the global society. Nitobe School faculty members are required not only to enhance their facilitation skills but to become role models of specialist and generalist, which mean they are expected to utilize their expertise and research experiences to instruct TBL/PBL style trans-graduate class. So, it is required to suggest a new idea to build new and rather unusual relationship between students and instructors, which would establish the method of TBL/PBL style class for graduate students from different graduate school.

Reference [12] concluded that "In order to improve such learning environment, we should promote professors and faculty to deeply respect and understand differences beyond their expertise. That is, the target of human resources that correspond to drastic change in international society and is possible through combined efforts from professors as well as graduate students." Therefore, Nitobe School faculty members are required to review their class organization to improve and develop their curriculum design as "check" stage in PDCA (Plan-Do-Check-Action) cycle. However, it is difficult to evaluate the learning outcomes in such a TBL/PBL style class. Thus, we adopt a questionnaire survey taken from both students and instructors to specify problems to be considered. We asked students their opinions about instructors' abilities and attitude. We also took similar questionnaire for instructors to compare both.

This paper will present a first study of a role of instructor in Nitobe School class, which can be considered as a sample of a TBL/PBL style class for trans-graduate school education. We will discuss the effect of instructor's attitudes and actions on student's motivation and discussion process. Instructors tried their own approach to supervise the student groups based on their experience. This paper aims to assess their teaching approaches according to the questionnaire for both students and instructors. Furthermore, we discuss the suitable number of instructors in one class. To instruct students and supervise group discussion in the Nitobe School classes, three or four instructors were assigned. We review and assess the assignment system to improve and establish the teaching method of TBL/PBL class for graduate education. The paper is designed as below. Section 2 details the background of Nitobe School and the framework of the fourth quarter of the school which we focus on. Section 3 shows the questionnaire survey and its result for both of students and instructors. The questionnaire questioned instructor's actions to investigate the effect of it on student's motivation. Discussion on the suitable number of instructors in the class is presented in section 4 . The number of instructors is one of key factor to establish effective and efficient method of TBL/PBL class for trans-graduate education. Section 5 is devoted to discussion and conclusion.

\section{Background}

The basic program of Nitobe School consists of four quarters in a yearlong as reported in [11]. Each quarter in eight weeks aims the students to improve their skill-set quarter by quarter. The objective of the first quarter is for students to learn tools and manners of group working with other students who are from different graduate schools to solve social problems. In the second quarter, students were asked to work as a group for the given global topic, and they were expected to find out a way to solve the global issue with supervisor's close guidance. In the third quarter, students were asked to tackle on given local problems by themselves, and they were expected to suggest unique ideas and solutions as qualified problem-solvers. In the fourth quarter, we let 
students to search for hidden-local problems, which cannot be obviously perceived as problems by people, present in the local society through fieldwork, and they were expected to identify and clarify new ill-defined problems.

\subsection{Framework of Problem-finding Course}

In this paper, we report the class held in 2015 academic year. The period of the quarter was held from December 1st on 2015 to February 2nd on 2016. A total of 43 students participated in the course and were divided into two classes, Tuesday class and Thursday class. Classes took place every week for eight times. Students were asked to find out the hidden-local problem and to explain the process of the finding though the fieldwork. They were divided into seven groups, and each group composed of 6-7 members while carefully considering the variety of the student's diversities. Fig. 1 shows the diversity of various background, including their graduate schools, gender, nationality and grade of the graduate school from the master course (MC1 to 2) doctoral course (DC1 to 3) and the professional school course (PC1 to 2) of all the enrolled students.
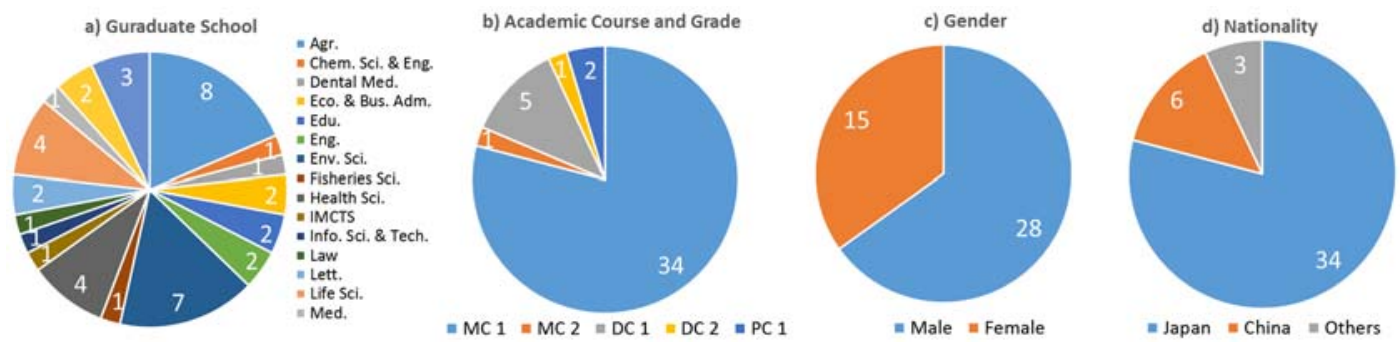

Fig. 1 The background of the completed students of the fourth quarter course $(n=43)$. Each graph denotes a) graduate school, b) academic course and grade, c) gender and d) nationality, respectively. The item "IMCTS" denotes Graduate School of International Media, Communication, and Tourism Studies and the item "Others" in Nationality includes students from Korea, Indonesia and Burkina Faso.

Each group was asked to play a role as a fictitious think-tank and each student in the group was expected to be a specialist of their research field, so that students were encouraged to respect each other in terms of each other's expertise and background during group discussions. The theme for their fictitious think-tank was set to be anything related to the ENERGY at local society. This rather broad theme let students to come up the topic from renewable energy problems at local, heat energy (such as hot spring), and energy to motivate high school students to study.

Table. 1 shows the tentative class schedule that was delivered during the first lecture guidance. Students were requested to complete the plan of fieldwork prior to the midterm presentation as the fourth week. Fieldwork was conducted at least one time during the course, and the plan of the fieldwork was presented as a format of poster presentation for the Nitobe mentors, alumni from Hokkaido University, in the midterm mentor exchange meeting [12]. The conclusion from each group was reported by the oral presentation in the last class of the quarter.

As for instructors, teaching and handling the class is a challenging subject since there are only few examples of such problem-finding class, especially for their teaching graduate students in home departments. Thus, instructors are also expected to gain experiences of 
finding/identifying problems from outside of their research field. The instructors of the Nitobe School classes are expected to generalize and utilize their research experience to instruct and supervise students who have different research background.

A unique point of the class was that at least one instructor stayed in each group in order to closely observe group activities, and at the same time facilitate their discussion if necessary. Each instructor supervised the group members based on their own teaching skills, teaching experience, and research expertise. Thus, different group might have received different feedbacks and suggestions.

Table. 1 The schedule of the class, which was shown in the guidance of the class.

\begin{tabular}{l|l} 
Week & The contents of the class \\
\hline 1st & Guidance and special lectures \\
\hline 2nd & Group discussion: decide the subject and fictitious client \\
\hline 3rd & Group discussion: decide the fieldwork place and plan \\
\hline 4th & Preparation for the poster and poster presentation (mentor exchange meeting) \\
\hline 5 th & Fieldwork \\
\hline 6th & Group discussion: review and summarize the result of fieldwork \\
\hline 7th & Group discussion: conclude and find the hidden local problem \\
\hline 8th & Preparation for the presentation and the final presentation
\end{tabular}

\section{Questionnaire Survey}

We carried out the questionnaire survey for both students and instructors to assess the effect of instructors' teaching actions and attitudes on the student's motivation and discussion. The questionnaires were taken place on the web survey using google form.

\subsection{Questionnaire for Students}

\subsubsection{Structure of the Questionnaire Survey and its Responders}

The purpose of the questionnaire for students is to elucidate their concerns for attitude and abilities of instructors after completion of eight weeks of the fourth quarter. The questionnaire was made available from March 8th to 18th, and the series of questions were written in English-Japanese bi-lingual expression. We show only English questions herein. The questions discussed in this paper were a part of the 23 questions, which can be found in and in accordance with their research purposes in [13] and [14]. 23 students out of the 43 participants in the fourth quarter answered the questionnaire and their background is represented in Fig. 2. The 23 responders might be cooperative students of Nitobe School, since the ratio of respondents is only around $50 \%$ of participants in the class. 

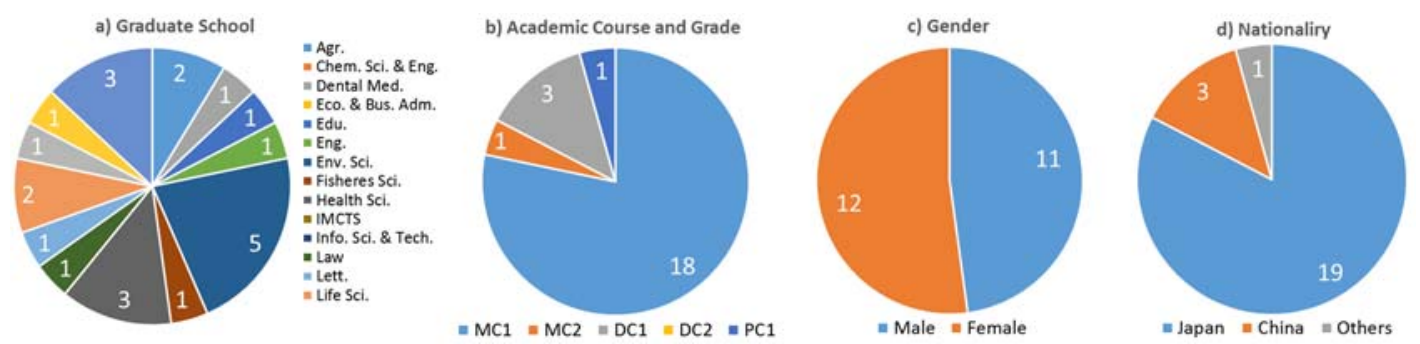

Fig. 2 The distribution of 23 respondents out of 43 participants. The items are same with Fig. 1.

The questions include general questions for TBL style class in whole Nitobe School (first to fourth quarters) and additional questions were included for the fourth quarter such as fieldwork. The questionnaire is presented in Table. 2. The questions asked the effect of instructor's supervision over group discussion and fieldwork.

\subsubsection{Results of the Questionnaire Survey}

The question 1 asked the number of instructors in the class. Answer a) is the present situation of the fourth quarter and 14 students were satisfied with the situation. The nine responded answer b), all instructors for all group is better. The question 2 asked the availability of instructors. All responses answered that all instructors were available even outside of class through e-mail or face-to-face meeting. The question 3 shows 19 students agreed that instructors gave important advices. Three students felt supervisor made the group discussion confusing and one responded the supervisor did not give any advice. With regard to question 4 from the questionnaire, 15 responded that supervisors encouraged students to use their background knowledge but seven said s/he did not motivate, while they felt that instructors gave some useful suggestions. In the question 5, 19 students felt that the supervisor participated in the group discussion like a student. In the active learning lecture, teachers usually act as a facilitator or advisor. In fact, this relationship can be considered as a colleague of students (equal partner to student) rather than a traditional teacher. For the result of question 6, 18 agreed with the instruction both of before and during fieldwork. Five responders were unsatisfied with supervisor's help during the fieldwork, especially two answered that supervisor did not help anything about fieldwork. The question 7 asked the action of the supervisor for the preparations of the midterm and final presentations. Almost all students (20) answered the supervisor checked at least the contents of the presentations, and they were positive responses. The question 8 asked the student's opinion about supervisor's attitude and their academic background. Majority of students (18) agreed with and expressed their respect to the teacher's professional background, while five students did not agree. It is noteworthy that most of students considered supervisor's comments were overall useful. The instructors are researchers at the same time, therefore their academic background can affect the comments and influence the group discussion.

Table. 2 The Questionnaire for students and its responses $(n=23)$. The instructor is called "supervisor" in the questionnaire. The word "NS" in the questions means "Nitobe School". 
Question 1. How do you think the number of Professors (Supervisors) in the classroom affected your learning experience considering the present scenario in NS?

a) One Professor for one group is better.

b) All Professors for all groups is better.

c) One Professor for the whole class is better.

d) The number of Professors does not affect the learning experience of students.

Question 2. Your supervisor was always available for you even outside of the class.

a) Supervisor was always available for any occasion.

b) Supervisor was available through emails but not for face-toface meeting.

c) Supervisor was available for meeting but was not through emails.

d) Supervisor was neither available for meeting nor email.

Question 3. Do you think your supervisor's comments in the group discussions were helpful to you?

a) Supervisor always gave important advices to improve our discussion.

b) Supervisor's comments made the group discussion more confusing.

c) Supervisor did not give any advice during group discussion.

d) Supervisor's advices were mostly not helpful.

Question 4. Do you think your supervisor always motivated you to enhance your specialty during group discussions considering background and diversity of your group?

a) Supervisor always motivated me to use my specialty and background.

b) Supervisor motivated me, but forced his/her opinion on me.

c) Supervisor did not motivate me, but s/he gave useful suggestions.

d) Supervisor did not pay respect and forced his/her opinion on me. 
Question 5. Did your supervisor participate in your group discussions just like a team member (equal to you)?

a) Supervisor was always present during discussions and contributed equally to students.

b) Supervisor listened to all discussions but did not participate as a member.

c) Supervisor did not listen or participate group discussions.

d) Supervisor acted like a traditional teacher (not equal to you).

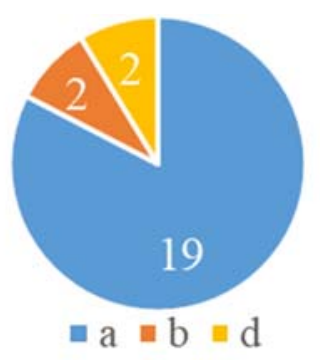

Question 6. What was the supervisor's role before and during the fieldwork?

a) The supervisor explained in detail about the fieldwork before and helped me during the field work as well.

b) The supervisor explained in detail about the fieldwork but did not help me during the fieldwork.

c) The supervisor did not explain anything before and during the fieldwork.

d) The supervisor did not explain anything before the fieldwork, but s/he helped during the fieldwork.

Question 7. Did the supervisor helped you with your preparation during the midterm and final presentations (contents and slides)?

a) Supervisor helped me with the contents and slides.

b) Supervisor helped me the contents but not the slides.

c) Supervisor did not help me with the contents but checked the slides.

d) Supervisor did not help me either contents or slides.

Question 8. Does your supervisor's academic background and experience affect your discussion and result?

a) Supervisor's academic background and experience influenced the team's thinking.

b) Supervisor did not use his/her academic background and experience during discussions, but gave useful comments.

c) Supervisor used his/her academic background and experience, but it did not improve the discussion.

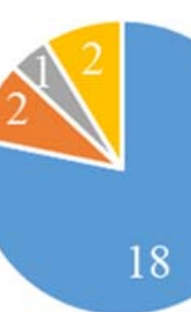

$\because \mathrm{a}=\mathrm{b} \equiv \mathrm{c} \equiv \mathrm{d}$

\section{8}

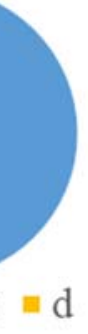

d) The supervisor's academic background and experience has no effect on us.

\subsection{Questionnaire for Instructors}

\subsubsection{Structure of the Questionnaire Survey and its responders}


The questionnaire to instructors was conducted from March 19th to 28th to elucidate what they thought and behaved to students in the course period. Almost questions were similar to the ones for students but asked from instructor's point of view (question 1 to 8). We further asked their teaching experience (question 9 and 10). The result can be compared to results from students in order to evaluate how their teaching actions and attitudes affect learning outcomes. The questionnaire is presented in Table. 3.

Totally five instructors including authors were assigned to the course and all of them completed the questionnaire. Three regular instructors belong to the Institute for the Advancement of Higher Education appointed to work in Nitobe School whose roles were to attend all Nitobe School classes and support a faculty member from outside of the school and each of them has different research background such as engineering, science and science communicator. Two instructors assigned in Tuesday and Thursday come from engineering and agriculture. Thus, four instructors are assigned in each class, Tuesday and Thursday. All the instructors are young in their 30's and 40's therefore their teaching experience and career are still unpracticed.

\subsubsection{Results of the Questionnaire Survey}

The number of respondents are just five while all assigned instructors answer the questionnaire. Since a personal respond can strongly influence the result of the questionnaire because of the small number of the respondents, we cannot analyze and discuss the tendency of the result in detail.

We pick up a few questions for instructors, which show large difference in their answers from students. The question 1 shows that one respondent answered that traditional style is better. There was no student to choose this answer. According to the question 5, three instructors did not act like a team member while 19 students answered the answer. The instructors observed the group discussion and gave comments as necessary.

The questions 9 and 10 are important to discuss the properties of instructors. The question 9 asks the experience of attending TBL class as an instructor or student and shows the three of five instructors have experience of attending TBL class. One has knowledge of teaching TBL class but no experience and one has no experience and knowledge. The question 10 shows all instructors have some experience of teaching both traditional or TBL class before joining the Nitobe School class. Two instructors have more than three years of teaching TBL class, so they are trainers of teaching to other three. Nitobe School also aims to give an opportunity for instructors to have an experience for teaching active learning class as FD. After teaching the class, they are expected to apply the experience to their home faculty to educate students. Therefore, we suggest to assign at least two instructors in the class. If a veteran and an unskilled instructor make a combination to teach such interdisciplinary class, it would be a good experience to the unskilled instructor as FD. To supervise a group discussion in higher education especially for interdisciplinary TBL/PBL class, instructors are required to utilize their academic background and experience to give useful comments to improve it. A young and unskilled instructor learns a way for teaching the TBL/PBL class and comprehensive point of view from a veteran partner. It seemed to be achieved in the fourth quarter course.

Table. 3 The questionnaire for instructors and their responses $(n=5)$. The result of the question 10 is shown in a bar graph because the question allows multiple selection. 


\begin{tabular}{|c|c|}
\hline Question & Response \\
\hline $\begin{array}{l}\text { Question 1. How do you think the number of Professors } \\
\text { (Supervisors) in the classroom affected students learning } \\
\text { experience considering the present curriculum in NS? } \\
\text { a) One Professor for one group is better (present situation). } \\
\text { b) All Professors for all groups is better. } \\
\text { c) One Professor for the whole class is better (traditional style). } \\
\text { d) The number of Professors does not affect the learning } \\
\text { experience of students. }\end{array}$ & $=\mathrm{a}$ \\
\hline $\begin{array}{l}\text { Question } 2 \text {. Were you available for your group even outside of } \\
\text { the class? } \\
\text { a) I was always available for any occasion. } \\
\text { b) I was available through emails but not for face-to-face } \\
\text { meeting. } \\
\text { c) I was available for meeting but was not through emails. } \\
\text { d) I was neither available for meeting nor on email. }\end{array}$ & \\
\hline $\begin{array}{l}\text { Question 3. How did your comments influence the group } \\
\text { discussion? } \\
\text { a) I could always give important advices to improve and control } \\
\text { the discussion. } \\
\text { b) My advices often resulted in the group to be confusing. } \\
\text { c) I did not give any advice during the discussion. } \\
\text { d) The group did not listen and accept my advices. }\end{array}$ & \\
\hline $\begin{array}{l}\text { Question } 4 \text {. Did you motivate the group members to enhance } \\
\text { their specialty during group discussions considering their own } \\
\text { background and diversity of your group? } \\
\text { a) I always motivated them to use their own specialty and } \\
\text { background. } \\
\text { b) I motivated them, but did not consider their specialty and } \\
\text { background. } \\
\text { c) I did not motivate the group members, but often gave useful } \\
\text { suggestions. } \\
\text { d) I treated all members alike. }\end{array}$ & $=\mathrm{a}=\mathrm{b}$ \\
\hline
\end{tabular}


Question 5. Did you participate in your group discussions just like a team member (equal to students)?

a) I was always present during discussions and contributed equally to students.

b) I listened to all discussions but did not participate as a member.

c) I did not listen or participate in group discussions.

d) I did not act like a team member.

Question 6. What was your role before and during the field trip?

a) I explained in detail about the field trip before and helped students during the field trip as well.

b) I explained in detail about the field trip but did not help students during the field trip.

c) I did not explain anything before and during the field trip.

d) I did not explain anything before the field trip, but helped during the field trip.

Question 7. Did you supervise students with their preparation during the midterm and final presentations (contents and slides)?

a) I supervised them with the contents and slides.

b) I supervised them with the contents but not the slides.

c) I did not supervise them with the contents but checked the slides.

d) I did not supervise them for contents or slides.

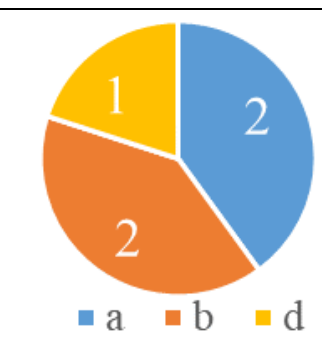

Question 8. Do you think your academic background and experience affect the group discussion and result?

a) My academic background and experience influenced the team's thinking.

b) I did not use my academic background and experience during discussions, but gave useful comments.

c) I used my academic background and experience, but it did not improve the discussion.

d) My academic background and experience have no effect on the group discussion. 
Question 9. Do you have experience to attend team based learning style class as a student?

a) Yes, I have many experience, and I know what student expect for teacher.

b) Yes, I have some experience.

c) No, but I know the role of teacher in a team based learning style class.

d) No, I never have any experience of team based learning.

Question 10. Do you have previous experience of teaching before? (Multiple selection is allowed)

a) I have experience more than 3 years for teaching team based learning.

b) I have some experience for teaching team based learning.

c) I have experience more of than 3 years for teaching traditional classes.

d) I have some experience for teaching traditional classes.

e) I have no experience for teaching.

\section{Discussion on the number of instructors in the class}

As we mentioned in the previous section, Nitobe School assigns several instructors in one class. In the fourth quarter, each instructor was assigned per group discussion. We review the way for teaching based on questionnaire results to evaluate a suitable number of instructors in one class. Effective and efficient teaching method of TBL/PBL class for graduate students by several instructors is not established yet since there are few examples of the class such as [15] and [16] in Japan. A case study of the number of instructors can be a significant report to establish the new teaching style. We analyzed the results of questionnaire for students. The result from question 1 suggests that the current system of Nitobe School, assigning a specific supervisor to a group, is satisfactory to majority of the students but nine students favored all instructors supervise for all group (answer b). This can relate to different actions and attitudes of every supervisor. Students who selected the answer b) may be unsatisfied with their supervisor by some reasons. We divide the respondents into two groups to analyze the relation of the tendency of the questions 3 to 8 according to the distribution of the answer of the question 1. Group A is composed of the students who selected the answer a) of the question 1 and group B is the students who selected the answer b). Table. 4 shows the distributions of the questions 3 to 8 among the original result which comes from Table. 2, group A and group B to compare the tendencies by them. The analysis the results of group A and B can suggest that the suitable number of teachers and method of the supervision of group discussion in TBL/PBL class for interdisciplinary graduate education.

Table. 4 The distributions of questions 3 to 8 among the original results from Table. $2(\mathrm{n}=23)$, group A $(n=14)$ and group $B(n=9)$. All the 23 respondents are divided into two groups according to the answer of the question 1 .

\begin{tabular}{|c|c|c|c|}
\hline Questions & Original & Group A & Group B \\
\hline
\end{tabular}




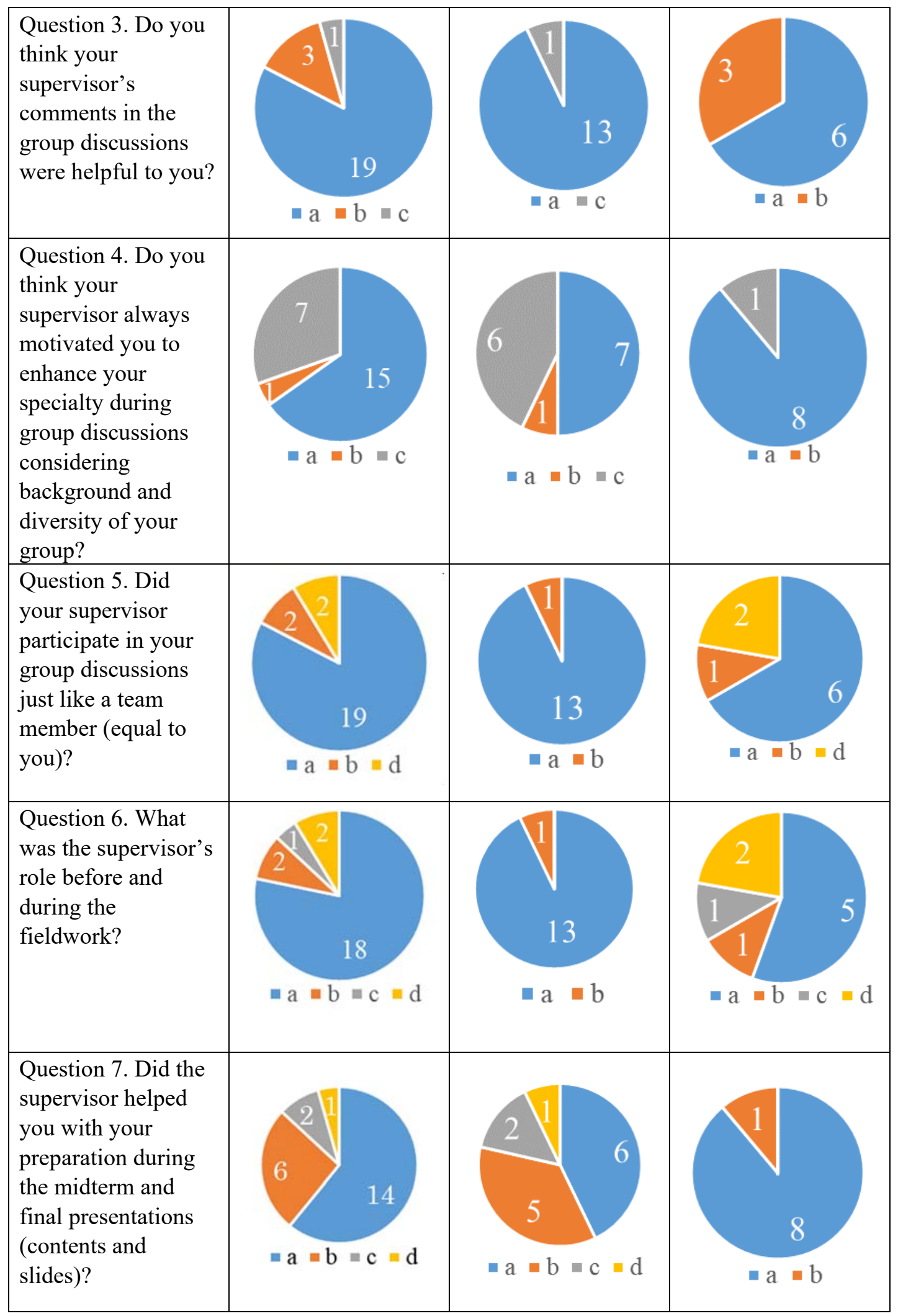




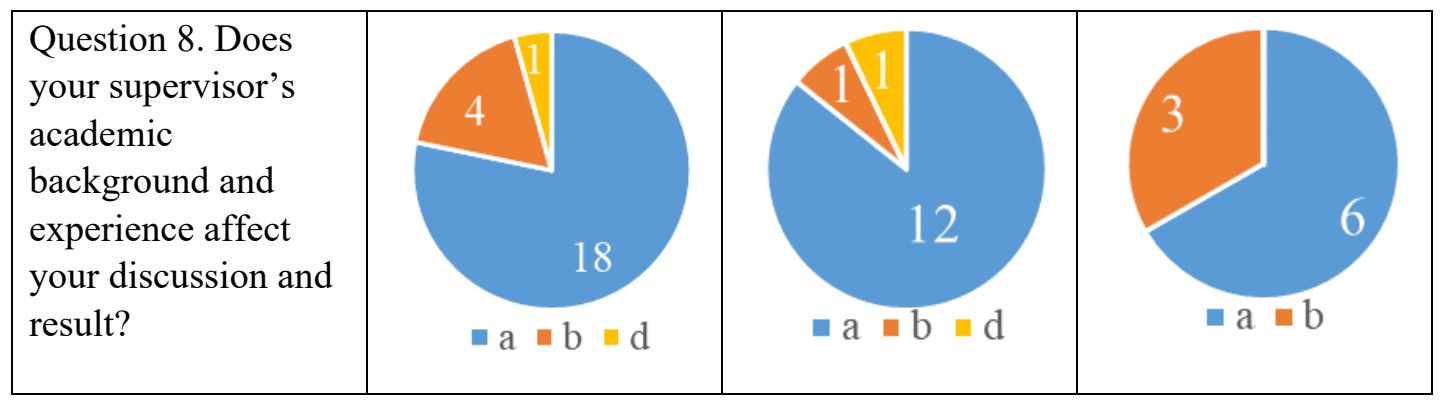

\subsection{Group A}

We firstly focus on the group A ( $\mathrm{n}=14)$. Questions 3, 5 and 6 suggest that almost students belonging group A are satisfied with their supervisors. They helped the group discussion with giving good advices and contributing equally to students, and helped fieldwork before and during. Question 4 suggests that the eight respondents including the answer b) recognized that supervisors motivated them and the six agreed their supervisors gave useful suggestions. These results obviously support the result of the question 1 . They appreciate supervisors' actions and attitudes are helpful to them and favor full supervision of their discussion. The question 7 shows the variety of answers in contrast to other questions. This result may suggest that students in group A do not care about helps for presentations. We will discuss the result comparing with group B in the subsection 4.3. The question 8 shows total 13 students out of 14 appreciate instructor's comments based on academic background and experience affect their discussion.

\subsection{Group B}

Next, we discuss the result of group B $(n=9)$. The students in group B seem to be unsatisfied with the current system of Nitobe School by some reasons. The trends of the results of these questions are different from the original results which are shown in Table. 2. Basically, the choice of answers a) to d) in each question correspond to satisfied to unsatisfied. The results of the questions 3,5 and 6 show that the ratios of a) are decreased compering with the original results. All the students who select the answer b) of the question 3 and the answer c), d) of the question 5 and 6 belong the group B. This result is obviously the reason that they were not satisfied with their supervisors. The results of the questions 4,7 and 8 , however, show different trends. The students were motivated and obtained useful comments based on supervisor's specialty during the discussion and helped for the preparation of the presentations at least the contents by the supervisor. Nevertheless, they favored all professors for all groups. This result may be interpreted that students refuse any disturbance by supervisor and actions as a teacher, although they need helps and advices in the class. In conclusion, students who favored all instructors for all group were unsatisfied with their supervisors and would need more advices and comments from other professors.

\subsection{Comparison between group $A$ and $B$}

To analyze the tendency of the group A and B, we compare with the results in Table. 4. The ratio of answer a) is decreased and the answers c) and d) are included to group A to B except for the question 7. This result clearly show the satisfactory of attitudes and actions of supervisors. In the case that a student is satisfied with the support from his/her supervisor, s/he may favor one instructor fully supervises the group work. On the other hand, if a student did not satisfy with 
his/her supervisor for some reasons, s/he favored the answer b) in the question 1 to obtain helps from other supervisors.

As for the question 7 in the group A, one student thought their supervisors did not help for their midterm and final presentations, and two students thought their supervisors did not help for the contents of the presentations while they favored one instructor supervises a group. On the other hand, the students in group B recognized their supervisor helped at least contents for their midterm and final presentations, even though they favored all instructors supervise all group. It is difficult to interpret and identify the reason for the results, but it may be a reason that three students who answered c) and d) and five students who answered b) in the question 7 in group A did not need any helps for the presentations by their supervisor, while the presentations are the outputs or outcomes of their learning process in the class. The result of question 7 may suggest that students do not care about help of their output by supervisors and intervention of it is not influenced their satisfaction. We need more investigations to identify the reason of such difference.

As a conclusion of this section, the answer of the question 1 can relate the actions and attitudes of instructors and satisfaction of students. A suitable number of instructors and the supervision of the group discussion in TBL/PBL class might depend on the attitudes and actions of instructors. To design a TBL/PBL class in higher education, the number of instructors can be decided based on their experience. If the instructors have enough experience to supervise TBL/PBL class, it can be less assigned, and vice versa. According to the result of the question 1 for students, we can say that students prefer to assign some instructors to supervise the group discussion in a TBL/PBL class. They seem to require many advices and helps during discussion. This result supports our suggestion in the previous section that at least two instructors should be assigned, from the student's point of view.

Unfortunately, the number of instructor in the course is yet just five, which is not enough to analyze in detail such like this section. To perform more in-depth analysis between the feedbacks from students and instructors, we will need to collect more data from the instructor's point of view.

\section{Conclusion}

We have investigated the effect of teacher's actions and attitudes on student's motivation and discussion in the problem-finding course in the new trans-graduate program of Hokkaido University, Nitobe School based on the questionnaire survey for both of students and instructors. Our approach to compare and analyze the feedbacks from both side of students and instructors enable us to assess the effect of instructor's actions on students' motivation and discussion process. Our final goal is to assess the learning outcomes of the class. It is, however, difficult to assess the learning outcomes directory to review the teaching method. Since the learning outcomes of the class may be affected by the motivation and process, our investigation can be a first step to achieve the goal. Furthermore, we have discussed a suitable number of the instructors in one class. While we could not make a conclusion for the discussion, we found that the trends of the answers are related to the level of satisfaction of instructors. The answers asking the actions and attitudes of supervisors are different between the group A and B. The comparison may suggest that the suitable number of instructors depends on the actions and attitudes of instructors to motive students but at least two instructors should be assigned to be a good supervision. While 
Nitobe School assigned several instructors equal to the number of the groups in the class, the school could reduce the number of the instructors if they have enough skills to encourage and motive students through the support of the group work.

As pointed out in the previous paper [13], there were few examples of a separate course of problem-finding strategy, hence the effective and efficient methods to instruction was not established. Since 2015 was the first year of the Nitobe School, each instructor tried their own approaches based on their experiences and knowledge. In the end, both of students and instructors had a good experience through taking the course. Instructors established their original approaches to supervise students so that students achieved to understand the importance of problem finding/identification.

Nitobe School gives new opportunities for faculty members to overview their research experience and knowledge from a general point of view to instruct trans-graduate school classes. This teaching opportunity is difficult to give in individual graduate school where ought to educate specialized knowledge and skills to nurture a specialist of the research field. Resent graduate school education is also demanded to cultivate a transferable skills and knowledge to play leading roles in the global society. Faculty members in Nitobe School face the demand and follow the PDCA cycle to develop new approaches toward education in high quality. The Nitobe School education can be considered as an experimental stage of FD. Faculty members would gain experience and share their trial and error to improve the education with each other. This is a challenging trial to a young faculty to overview their research experience and knowledge from a general point of view. The trained instructor will be back to his/her faculty and give a better education to nurture a graduate student to be a specialist with a comprehensive and applicable skills and knowledge.

In this paper, we focused on the fourth quarter of the Nitobe School program. We will perform analogous approaches for all quarters and make the curriculum design to collect effective survey data. Higher education, especially for graduate school education, needs such collection of cases to establish a manual or handbook on education for graduate students. For example, the book [17] reported a result of questionnaire survey on the faculty misconduct in graduate education. Our investigation is one of the cases for the trans-graduate school education in TBL/PBL style class.

\section{Acknowledgement}

The authors acknowledge Associate Professor M. Namba for teaching the classes in Nitobe School and Prof. H. Haga for organization of the class design of the fourth quarter. This work is supported by "Top Global University Project" by the Ministry of Education, Culture, Sports, Science and Technology, Japan and Nitobe School at Hokkaido University, Sapporo, Japan.

\section{References}

[1] National Institution for Academic Degrees and University Evaluation, "Overview of the Quality Assurance System in Higher Education: JAPAN (second edition)," 2014. 
[Online].

Available: http://www.niad.ac.jp/english/overview_jp_e_ver2.pdf. [Accessed 24th April 2017].

[2] Organisation for Economic Co-operation and Development, "The definition and selection of key competencies," 2005. [Online].

Available: https://www.oecd.org/pisa/35070367.pdf. [Accessed 1st May 2017].

[3] Central Council for Education, Ministry of Education, Culture, Sports, Science and Technology-Japan (MEXT), "Toward construction of the undergraduate education," 2008. [Online].

Available:

http://www.mext.go.jp/component/b_menu/shingi/toushin/_icsFiles/afieldfile/2008 /12/26/1217067_001.pdf. [Accessed 20th April 2017].

[4] Ministry of Economy, Trade and Industry-Japan, "Fundamental Competencies for Working Persons (in Japanese)," 2006. [Online].

Available: http://www.meti.go.jp/policy/kisoryoku/. [Accessed 20th April 2017].

[5] Central Council for Education, MEXT, "Graduate School Education in the New Age -Towards the Development of Internationally Attractive Graduate School Educaiton(in Japanese)," 59 2005. [Online].

Available:

http://www.mext.go.jp/b_menu/shingi/chukyo/chukyo0/toushin/05090501/all.pdf. [Accessed 20th April 2017].

[6] Central Council for Education, MEXT, "Graduate School Education in a Globalized Society -To Make Graduate School Graduates Active in Various Fields of the World(in Japanese)," 311 2011. [Online].

Available:

http://www.mext.go.jp/component/b_menu/shingi/toushin/_icsFiles/afieldfile/2011 /03/04/1301932_01.pdf. [Accessed 20th April 2017].

[7] Center for Development and Support of Higher Education, Rikkyo University, "Reports on Development of Higher Education, No. 18 "Invitation to Supervision in Graduate School: Six Foreign Handbooks for Supervisors" (in Japanese)," 2013. [Online]. Available: http://www.rikkyo.ac.jp/about/activities/fd/qo9edr0000005dbr- 
att/No.18.pdf. [Accessed 1st May 2017].

[8] Center for Development and Support of Higher Education, Rikkyo University, "Reports on Development of Higher Education No. 23 "Interview; Way to the Ph.D. in Foreign Universities: Experience of Nine Rikkyo Faculties" (in Japanese)," 2015. [Online]. Available: http://www.rikkyo.ac.jp/about/activities/fd/qo9edr0000005dbratt/No.23.pdf. [Accessed 1st May 2017].

[9] Hokkaido University, "Future Strategy for the 150th Anniversary of Hokkaido University (in Japanese and English)," 2014. [Online].

Available: https://www.global.hokudai.ac.jp/wp-content/uploads/2014/09/FutureStrategy-for-the-150th-Anniversary-of-Hokkaido-University-2014.8-bilingual1.pdf. [Accessed 1st May 2017].

[10] Nitobe School, Hokkaido Univerisy, [Online]. Available: https://nitobe-school.academic.hokudai.ac.jp/en/. [Accessed 20th April 2017].

[11] Japan Society for the Promotion of Science, "Top Global University Project (in Japanese)," 2014. [Online]. Available: http://www.jsps.go.jp/j-sgu/gaiyou.html. [Accessed 20th April 2017].

[12] Y. Yamanaka, M. Shimamura, "A Trans-graduate-school Education Program Awaking Competencies to Graduate Students in Research University - The concept of Nitobe School program in Hokkaido University," International Journal of Institutional Research and Management, Vol. 1, No. 2, pp. 37-53, 2017.

[13] A. A. Ravankar, S. Imai, M. Shimamura, G. Chiba and T. E. Takasuka, "Problembased Learning and Problem Finding Among University Graduate Students," J. Higher Education and Lifelong Learning, vol. 24, pp. 9-20, 2017.

[14] M. Shimamura, S. Imai, A. A. Ravankar, Y. Yamanaka, "How Does the English Ability of the Student Change through the Postgraduate Education? The Case Report of "Nitobe School Program" in Hokkaido University"," 2016 5th IIAI International Congress on Advanced Applied Informatics (IIAI-AAI), p. 574, 2016.

[15] The University of Tokyo, "i. school," [Online]. Available: http://ischool.or.jp/. [Accessed 10th Sptember 2017].

[16] M. Ichiki, M. Suzuki, S. Mine, H. Sautoh, K. Sugawara, Y. Kanazawa and R. 
Naganawa, "Recent program of AIST Innovation School For the future innovation leader in global society," 2017 6th IIAI International Congress on Advanced Applied Informatics (IIAI-AAI), 2017.

[17] J. M. Braxton, E. Proper , A. E. Bayer, Professors Behaving Badly: Faculty Misconduct in Graduate Education, Baltimore: The Johns Hpkins University Press, 2011. 